\title{
Bicycle Freewheeling with Air Drag as a Physics Experiment
}

Paul Janssen and Ewald Janssens

Citation: The Physics Teacher 53, 25 (2015); doi: 10.1119/1.4904237

View online: http://dx.doi.org/10.1119/1.4904237

View Table of Contents: http://scitation.aip.org/content/aapt/journal/tpt/53/1?ver=pdfcov

Published by the American Association of Physics Teachers

\section{Articles you may be interested in}

Fizeau's "aether-drag" experiment in the undergraduate laboratory

Am. J. Phys. 80, 497 (2012); 10.1119/1.3690117

The Physics of Bicycle Stability bicycle.tudelft.nl/stablebicycle

Phys. Teach. 50, 125 (2012); 10.1119/1.3677299

iOS physics learning apps (for Apple products iPhone, iPod Touch, iPad)

Phys. Teach. 50, 61 (2012); 10.1119/1.3670096

Teaching laser physics by experiments

Am. J. Phys. 79, 85 (2011); 10.1119/1.3488984

The Physics of the Imploding Can Experiment

Phys. Teach. 48, 289 (2010); 10.1119/1.3393054

$\therefore$ LABQUEST 2

The Most Powerful and

Versatile Data Logger for

Physics Education

www.vernier.com/labquest

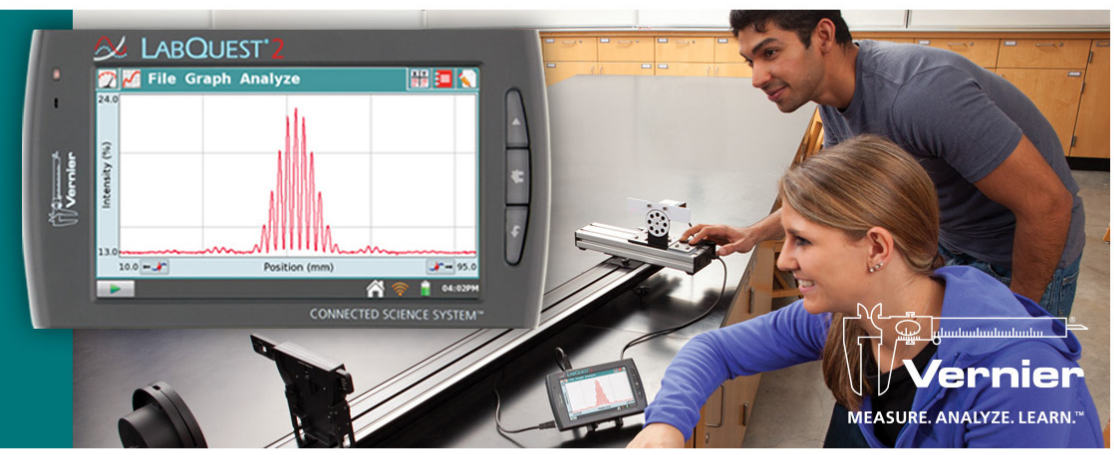




\section{Bicycle Freewheeling with Air Drag as a Physics Experiment}

Paul Janssen and Ewald Janssens, KU Leuven, Department of Physics and Astronomy, Belgium

$\mathrm{T}$ o familiarize first-year students with the important ingredients of a physics experiment, we offer them a project close to their daily life: measuring the effect of air resistance on a bicycle. Experiments are done with a bicycle freewheeling on a downhill slope. The data are compared with equations of motions corresponding to different models for the air resistance on a moving object. The relevant parameters are extracted from the data and the validity of the different models is be discussed. Finally, findings are presented in a poster session.

\section{Air drag}

The subject of air drag or air resistance has been used in undergraduate labs many times, both for spherical ${ }^{1-3}$ and cylindrical ${ }^{4-5}$ objects, and even for irregular shapes such as the human body. ${ }^{6}$ All of these deal with falling bodies for which the only friction is due to the surrounding air. When one rides a bicycle, in addition to air drag, rolling friction also has to be taken into account. ${ }^{7,8}$ Studies have demonstrated that different cycling conditions, such as different masses, cross sections, and tire pressure changes, allow one to isolate variations in aerodynamic and rolling resistances. ${ }^{9}$ We provide our undergraduate students, along with their course handbook, the short, descriptive article by L. J. F. Hermans in Europhysics News. ${ }^{10}$

Accordingly, the frictional force is written as the sum of the rolling friction and the drag force. The rolling friction is taken proportional to the normal component of the weight and is written as $m g \cos (\theta) C_{\mathrm{R}}$, where $\theta$ is the slope angle. The drag force is written as $1 / 2 \rho A v^{2} C_{\mathrm{D}},{ }^{1,7,8}$ The symbols have their usual meaning: $m$ is the total mass of the moving object, $v$ is the speed, $g$ is the acceleration of gravity, $\rho$ is the density of air, and $A$ is the exposed surface. $C_{\mathrm{R}}$ and $C_{\mathrm{D}}$ are dimensionless coefficients. However, as $1 / 2 \rho A v^{2} C_{\mathrm{D}}$ is not derived for the irregular shape of a cyclist and as $A$ is somewhat difficult to define in this case, we introduce the coefficient $D$ [expressed in $\mathrm{N} /$ $\left.(\mathrm{m} / \mathrm{s})^{2}\right]$ and write the drag term simply as $D v^{2}$. This represents a quadratic model, which will be one of our test models.

As an alternative test model, a resistance force equal to the sum of the rolling force and a term proportional to $v$ is proposed: $m g \cos (\theta) C_{\mathrm{R}}+k v$. This corresponds to the Stokes' model students find in their textbook for low Reynolds numbers, if the coefficient $k$ [expressed in $\mathrm{N} /(\mathrm{m} / \mathrm{s})$ ] absorbs all the factors in the normal expression for the Stoke's law.

Approximating the Reynolds number by $R=\rho L v / \eta$, with $\rho$ the air density, $L$ a typical length scale of the bicycle, and $\eta$ the viscosity, one obtains $R \approx 10^{5}$ for $v=1 \mathrm{~m} / \mathrm{s}$. Since $R » 1$ (large Reynolds number regime) the quadratic model for the air resistance is expected to give a better description than a Stokes' type of air drag. ${ }^{3}$ One task for the students is to find out if a clear discrimination between these two models can be made based on their measurements, a task that will turn out to be not as straightforward as one may anticipate.

\section{Performing the experiment}

To carry out the experiment, a student on a bicycle rolls down a slope, without using the pedals or brakes, with or without initial speed. The bike is equipped with a small magnet on one of the spokes and a reed switch on the frame. The time of each closure of this switch is sent to a memory chip. The measuring unit is stored in a box installed on the luggage carrier of the bike. Full details are given in an electronic addendum. ${ }^{11}$ At the end of the ride, the cyclist goes to the teaching assistant, who uses a LabVIEW virtual instrument ${ }^{12}$ to transfer the recorded data to a computer using a USB cable and resets the clock in the measurement device so that a next experiment can then be undertaken.

It is important that the slope is a stretch of road descending under a fairly constant angle $\theta$. In our case the total length of the stretch is about $300 \mathrm{~m}$ and it has a reasonably constantslope angle $\theta \approx 2.0^{\circ} \pm 0.3^{\circ}$, corresponding to a slope of $\tan \theta \approx$ $3.5 \% \pm 0.5 \%$.

All measurements are done during the first session of the project. The students work in small groups of three or four students. Several groups can carry out the experiments in parallel. Aside from the teaching assistant, a technician is also present at this session to install the mobile measurement device on the bicycles. Recording the necessary data takes a few hours at maximum. The students are themselves fully responsible for the completeness of their data. The table of times collected during a "run" can be used to construct an $(x, t)$ graph, with $x$ expressed in wheel perimeters. After measuring the diameter of the wheel, $x$ is easily converted into a distance in meters. Here enter some of the important goals of a firstyear physics laboratory. The students have to think about accuracy and precision. How accurate is this conversion? How important is this accuracy? Other points of concern are, as mentioned before, the slope angle and its constancy, the effect of local imperfections of the stretch, variations in wind (both speed and direction), mass, and perhaps an estimate of the cross section. A variety of measuring devices (including an anemometer and a digital level meter) is put at the students' disposal. The students are advised to repeat the measurement under similar conditions to get a feeling for the precision.

To obtain a deeper insight in the role of the different variables, each group is asked to make variations in their procedures, for example, by trying different masses (more than one person on a bike), using different cross sections (taking along 
an opened umbrella), starting with different initial speeds, and riding with underinflated tires. A particularly interesting but somewhat difficult variation is to go uphill instead of downhill. This requires careful revision of the math done.

\section{Data analysis}

In the following sessions, data are analyzed. First, the equation of motion has to be solved for both test models. Define an $x$-axis along the slope and pointing downward. Let the initial speed be $v_{0}$ and choose $x(t=0)=0$.

For the linear model, obtaining the solution is straightforward and students are expected to do the math on their own. It is summarized in Table I.

Table I. Equation of motion and solutions for the "linear" model.

\begin{tabular}{|l|l|}
\hline $\begin{array}{l}\text { Equation } \\
\text { of motion }\end{array}$ & $m \frac{d v_{x}}{d t}=m g \sin \theta-C_{\mathrm{R}} m g \cos \theta-k v_{x}(t)$ \\
\hline Abbreviations & $\tau=\frac{m}{k}$ \\
$v_{\mathrm{E}}=\frac{m g}{k}\left(\sin \theta-C_{\mathrm{R}} \cos \theta\right)$ \\
\hline Speed & $v_{x}(t)=\left(v_{0}-v_{\mathrm{E}}\right) e^{-t / \tau}+v_{\mathrm{E}}$ \\
\hline Distance & $x(t)=v_{\mathrm{E}} t+\tau\left(v_{0}-v_{\mathrm{E}}\right)\left(1-e^{-t / \tau}\right)$ \\
\hline
\end{tabular}

As can be seen from (4), the speed evolves with time towards the constant $v_{\mathrm{E}}$, the terminal speed at which the friction balances the component of gravity along the slope. Solving the quadratic case is somewhat harder and surpasses the present capabilities of the students. Therefore, the solutions are simply given (see Table II). Again, $v_{\mathrm{E}}$ is the terminal speed.

Table II. Equation of motion and solutions for the "quadratic" model.

\begin{tabular}{|l|l|}
\hline $\begin{array}{l}\text { Equation } \\
\text { of motion }\end{array}$ & $m \frac{d v_{x}}{d t}=m g \sin \theta-C_{\mathrm{R}} m g \cos \theta-D v_{x}^{2}(t)$ \\
\hline Abbreviations & $v_{\mathrm{E}}=\sqrt{\frac{m g\left(\sin \theta-C_{\mathrm{R}} \cos \theta\right)}{D}}$ \\
& $\begin{array}{l}\tau=\frac{m}{D v_{\mathrm{E}}} \\
\\
c=\frac{v_{\mathrm{E}}+v_{0}}{v_{\mathrm{E}}-v_{0}}\end{array}$ \\
\hline Speed & $v_{x}(t)=v_{\mathrm{E}}\left[\frac{c e^{2 t / \tau}-1}{c e^{2 t / \tau}+1}\right]$ \\
\hline Distance & $x(t)=v_{\mathrm{E}} \tau \ln \left[\frac{1+c e^{2 t / \tau}}{(1+c) e^{t / \tau}}\right]$ \\
\hline
\end{tabular}

A typical measurement is shown in Fig. 1. It displays the distance traveled as a function of time for a descent with zero

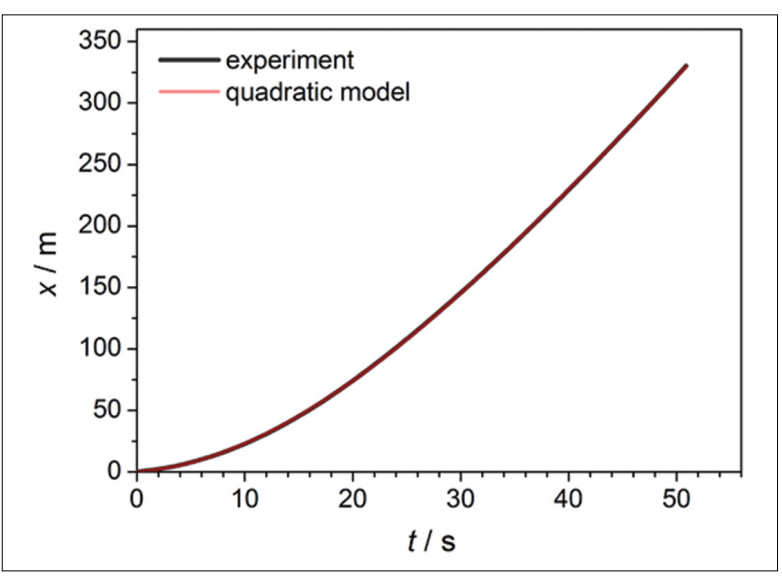

Fig. 1. A typical result for a descent with zero initial speed. As the speed approaches its terminal value, the curve becomes a straight line.

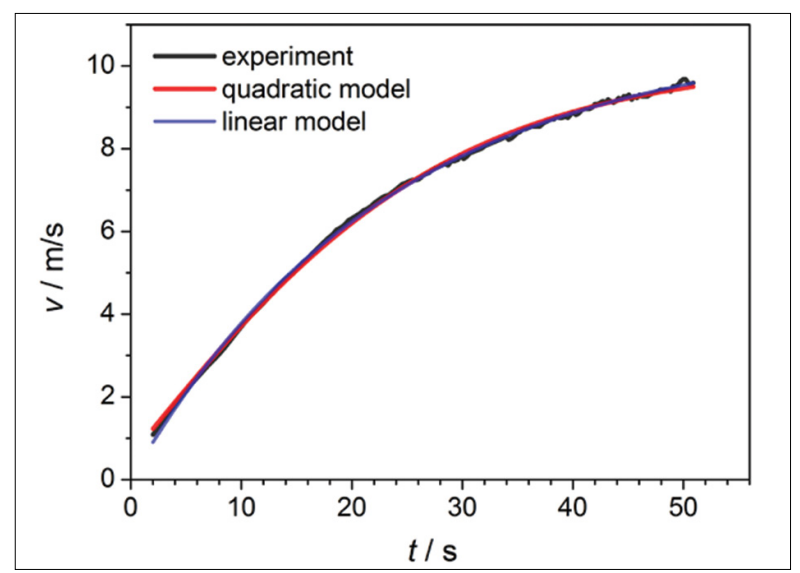

Fig. 2. Speed vs time (in black) compared to the linear [Eq. (4)] and quadratic [Eq. (10)] models.

initial speed. As can be seen, the line on the graph becomes more of a straight line as the speed approaches its terminal value. Such a distance-versus-time graph tends to mask the imperfections of the measurement. These are much more visible in a speed-versus-time graph, as in Fig. 2. Here the speed was obtained by making a simple numerical differentiation of the data from Fig. 1, i.e., $v_{i+1}$ is taken as $\left(x_{i+1}-x_{i}\right) /\left(t_{i+1}-t_{i}\right)$, with the index $i$ running over the data.

Figure 2 shows that the speed increases with time and approaches a terminal value. The expressions from Eqs. (4) and (10) are fitted to the black curve in Fig. 2. Our students use the software package Graphical Analysis from Vernier Software. ${ }^{13}$ Also Eq. (11) was used to fit the data on Fig. 1 [in principle also Eq. (5), but this is indistinguishable from Eq. (11) and is omitted for clarity]. Note the highest speed actually reached during the descent is slightly lower than the fitted "terminal speed": to reach the latter, a longer runway would be needed. But it is not so easy to find a long smooth stretch with a constant slope angle.

As can be seen immediately in Fig. 2, it is nearly impossible with a single measurement to tell which of the two models is the better one. Both fits visually nearly coincide and the root mean square error of the residuals with respect to the 
experimental data is almost equal.

The reason why one cannot distinguish between both test models using a single experiment is the "flexibility" of the fitting curves related to the presence of two fitting parameters $\left(v_{\mathrm{E}}, \tau\right)$. Moreover, it should also be remarked that experimental conditions are not perfect: the slope angle is not as constant as one would like, there are local imperfections on the stretch, wind may influence the motion (the equation of motion is in principle only valid if there is no wind), and turbulences can be important. Because of this even a "theoretically correct" model will not fit the data perfectly.

Therefore, the students are asked to make multiple measurements on the same slope for different initial velocities. For each measurement they should extract the initial acceleration, $a_{0}$ [the tangent of the $v(t)$ curve at $t=0$ ]. If the

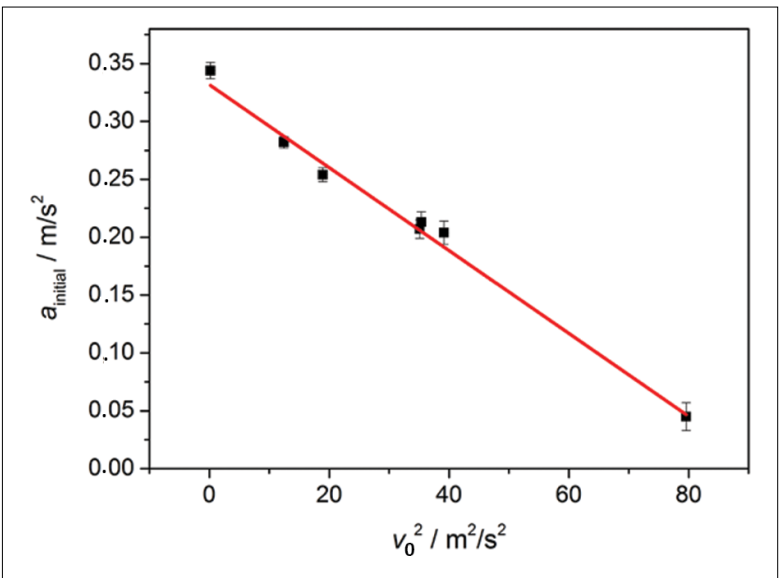

Fig. 3. Plot of $a_{\text {initial }}$ as function of $v_{0}^{2}$. According to Eq. (6) a linear relation should be obtained, which is indeed the case.

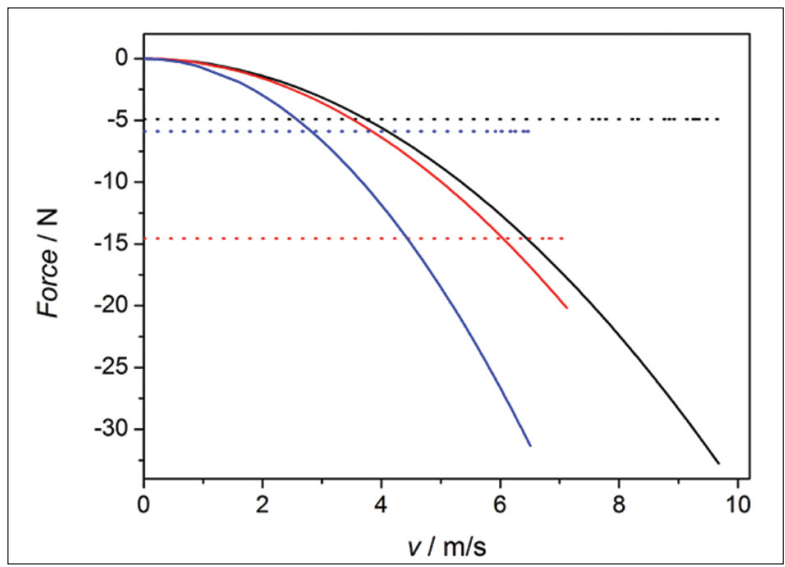

Fig. 4. Frictional forces acting on the bicycle as function of the speed. Dotted and full lines represent the rolling friction and the drag force, respectively. The black line is the reference measurement, and the red and blue lines represent experiments with underinflated tires and students holding an umbrella behind the bicycle, respectively. The curves end in the obtained terminal speed, where the total force acting on the bicycle is zero.
Table III. Results from curve fitting using the quadratic model for experiments carried out under different conditions. $\tau$ and $v_{E}$ are fitting parameters, $C_{R}$ and $D$ are derived using Eqs. (7) and (8). $C_{D}$ is obtained from $D$ using $D=1 / 2 \rho A C_{D}$.

\begin{tabular}{|c|c|c|}
\hline Reference measurement & $\begin{array}{l}\text { Larger cross section } \\
\text { (with open umbrella) }\end{array}$ & Underinflated tires \\
\hline $\begin{array}{l}\tau=(30 \pm 1) \mathrm{s} \\
v_{\mathrm{E}}=(10.1 \pm 0.2) \mathrm{m} / \mathrm{s}\end{array}$ & $\begin{array}{l}\tau=(21 \pm 1) \mathrm{s} \\
v_{\mathrm{E}}=(6.5 \pm 0.2) \mathrm{m} / \mathrm{s}\end{array}$ & $\begin{array}{l}\tau=(34 \pm 1) \mathrm{s} \\
\mathrm{v}_{\mathrm{E}}=(7.5 \pm 0.2) \mathrm{m} / \mathrm{s}\end{array}$ \\
\hline $\begin{array}{l}C_{\mathrm{R}}=(0.005 \pm 0.002) \\
D=(0.35 \pm 0.02) \mathrm{Ns}^{2} / \mathrm{m}^{2} \\
C_{\mathrm{D}}=(0.85 \pm 0.13)\end{array}$ & $\begin{array}{l}C_{\mathrm{R}}=(0.006 \pm 0.004) \\
D=(0.74 \pm 0.02) \mathrm{Ns}^{2} / \mathrm{m}^{2} \\
C_{\mathrm{D}}=(0.91 \pm 0.14)\end{array}$ & $\begin{array}{l}C_{\mathrm{R}}=(0.016 \pm 0.004) \\
D=(0.40 \pm 0.02) \mathrm{Ns}^{2} / \mathrm{m}^{2} \\
C_{\mathrm{D}}=(1.04 \pm 0.16)\end{array}$ \\
\hline
\end{tabular}

starting position is the same, these $a_{0}$ values are extracted for the same slope angle $\theta$, and thus the uncertainty on $\theta$ is removed. Plotting $a_{0}$ as a function of $v(t=0)$ should according to Eq. (1) provide a linear relation with slope $-k / m$ and intercept $g \sin \theta-g C_{r} \cos \theta$. Equation (6) gives a linear relation when plotting $a_{0}$ as a function of $v^{2}$. As is illustrated in Fig. 3, this experiment demonstrates that the quadratic model [Eq. (6)] is a good description.

To illustrate the kind of information students could obtain by carrying out experiments under different conditions, the results of three experiments are presented in Table III. It concerns a reference measurement, a measurement with an enhanced cross section (an open umbrella was held behind the student's back), and an experiment with underinflated tires ( 0.8 bar gauge pressure instead of $2.5 \mathrm{bar}$ in the reference measurement). Although the values of the parameters obtained are anecdotal, they illustrate the order of magnitude.

The results are in line with expectations: although different values for $v_{\mathrm{E}}$ are obtained under different conditions, they all have, within the error bars, the same value for $C_{\mathrm{D}}$. The parameter $D$ thus indeed scales with the cross section.

The values in Table III are in line with $C_{\mathrm{D}}$ and $C_{\mathrm{R}}$ values reported by Lim et al. ${ }^{9}$ The value of $C_{\mathrm{R}}$ must be between zero and $\tan \theta=0.035$; otherwise, the bicycle would not begin to roll in an experiment with zero initial speed. The large uncertainty on $C_{\mathrm{R}}$ is related to the uncertainty on the slope angle $\theta\left(\Delta \theta=0.3^{\circ}\right)$ because the fitting procedure only provides a value for $\sin \theta-C_{\mathrm{R}} \cos \theta$. But what clearly can be seen is that the rolling resistance increases in the case of underinflated tires. The larger cross section has, within the obtained accuracy, no influence on $C_{\mathrm{R}}$. To graphically illustrate the forces that act on the cyclist along the stretch, the rolling resistance and the air drag are plotted in Fig. 4 as a function of speed for the three experiments in Table III.

\section{Presentation of the results and discussion}

After working everything out properly, the students have to make a report. This must be done in the form of a poster. The tutors give advice on how to make a decent poster. They help the students to select the presented material and give tips to make full use of the two-way communication between the presenter and the audience-an extremely useful exercise! 
Look What's in

The Physics Store!

Preconceptions

\section{in Mechanics}

This second edition of Charles Camp and John J. Clement's book contains a set of 24 innovative lessons and laboratories in mechanics for high school physics classrooms that was developed by a team of teachers and science education researchers. Research has shown that certain student preconceptions conflict with current physical theories and seem to resist change when using traditional instructional techniques. This book provides a set of lessons that are aimed specifically at these particularly troublesome areas: Normal Forces, Friction, Newton's Third Law, Relative Motion, Gravity, Inertia, and Tension. The lessons can be used to supplement any course that includes mechanics. Each unit contains detailed step-by-step lesson plans, homework and test problems, as well as background information on common student misconceptions, an overall integrated teaching strategy, and key aspects of the targeted core concepts. A CD of all duplication materials is included.

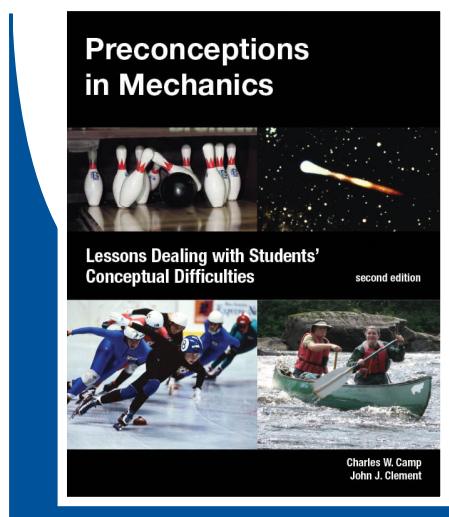

\section{Order yours now at www.aapt.org/store}

At the end, a poster session is organized which staff members of the department are invited to attend. Besides learning a lot from questions and comments, students gain a muchneeded element in their education: signs of interest in what they are doing.

\section{Acknowledgment}

The authors are greatly indebted to Mr. Ph. De Greef, KU Leuven, Dept. of Physics and Astronomy, for designing and constructing the measurement unit.

\section{References}

1. M. S. Greenwood, C. Hanna and J. W. Milton, "Air resistance acting on a sphere: Numerical analysis, strobe photographs, and videotapes," Phys. Teach. 24, 153-159 (March 1986).

2. P. Gluck, "Air resistance on falling balls and balloons," Phys. Teach. 41, 178-180 (March 2003).

3. K. Takahashi and D. Thompson, "Measuring air resistance in a computerized laboratory," Am. J. Phys. 67, 709 (Aug. 1999).

4. N. E. Derby, R. G. Fuller, and P. W. Gronseth, "The ubiquitous coffee filter," Phys. Teach. 35, 168-171 (March 1997)

5. R. A. Young, "Improving the data analysis for falling coffee filters," Phys. Teach. 39, 398 (Oct. 2001).

6. G. Wagner and R. Wood, "'Skydiver survives death plunge' (and the physics that helped)," Phys. Teach. 34, 543-545 (Dec. 1996).

7. P. E. di Prampero, G. Cortili, P. Mognoni, and F. Saibene, "Equation of motion of a cyclist," J. Appl. Physiol. 47, 201 (July 1979).

8. B. L. Hannas and J. E. Goff, "Model of the 2003 Tour de France," Am. J. Phys. 72, 575 (May 2004).

9. A. C. Lim, E. P. Homestead, A.G. Edwards, T. C. Carver, R. Kram, W. C. Byrnes, "Measuring changes in aerodynamic/ rolling resistances by cycle mounted power meters," Med. Sci. Sport Exer. 42, 853 (May 2011).

10. L. J. F. Hermans, "Physics in daily life: Drag 'n' roll," Europhys. News 35, 130, (July/Aug. 2004).

11. See supplementary material at TPT Online, http://dx.doi.org/ $10.1119 / 1.4904237$ for details on the electronics of the measurement unit.

12. http://www.ni.com/labview/.

13. http://www.vernier.com/products/software/ga/.

Paul Janssen is a retired professor at the KU Leuven, working in the Semiconductor Physics Laboratory, where he was conducting research in the magnetic resonance field using medium to high magnetic fields in combination with far-infrared lasers. He is vividly interested in educational work and chaired physics courses in many science directions. Paul.Janssen@fys.kuleuven.be

Ewald Janssens is associate professor of physics at the Laboratory of Solid State Physics and Magnetism, KU Leuven, Belgium. His research interests include electronic, structural, and magnetic properties of small atomic clusters studied with mass spectrometric and laser spectroscopic techniques. He also teaches an introductory physics practical course for bachelor students.

ewald.janssens@fys.kuleuven.be 